\title{
Synergistic 3D and 2D Imaging of Unique Extraterrestrial Samples for Curation, Sectioning, and Analysis.
}

\author{
Denton S. Ebel ${ }^{1,2}$, Jon M. Friedrich ${ }^{1,3}$, Shawn W. Wallace ${ }^{1}$, Ellen J. Crapster-Pregont ${ }^{1,2}$, and Amanda J. \\ White ${ }^{1}$. \\ 1. Dept. of Earth and Planetary Sciences, American Museum of Natural History, New York, NY 10024. \\ 2. Lamont-Doherty Earth Observatory, Columbia University, Palisades, NY 10964. \\ 3. Dept. of Chemistry, Fordham University, Bronx, NY 10458.
}

Extraterrestrial samples provide direct evidence of nucleosynthesis, interstellar dust composition, early solar system chemistry, and the origin of planets, asteroids and comets. Scientific collections of extraterrestrial material in museums and research laboratories continue to grow. It is increasingly important that collections incorporate digital data derived from their specimens. High value subsamples from the meteorite collection at the American Museum of Natural History have been imaged with synchrotron computed microtomography (S-CT) [1-5], and more recently with an in-house MicroCT scanner [6-9]. The 3D data can be used to guide physical cutting of samples to intersect specific features prior to preparation of polished petrographic sections and 2D electron beam analytical work [4]. Thus CT informs cutting, and preserves information about samples above and below the 2D plane of $\mathrm{x}$-ray energy dispersive (EDS) and wavelength dispersive spectrometric (WDS) mapping and quantitative analysis. Museum archives allow researchers who borrow imaged samples to skip many laborious steps in sample characterization, and mitigate material loss, handling, and x-ray damage hazards to the samples. Combined 3D and 2D data sets are also useful for public outreach and engagement. Development and implementation of these scientific and educational capabilities is in its infancy.

CT has been considered previously as a screening or curation tool for extraterrestrial samples [9-12], and was used to screen samples returned by the Hayabusa mission [13]. Commercial MicroCT systems informed sectioning of the Kobe (CK4) meteorite fall of 1999 for dissemination [12], and initial analysis of the Sutter's Mill CM chondrite breccia fall of April 2012 [6-8]. Synchrotron beamtime is not readily obtainable, but current advances in MicroCT have allowed institutions to acquire instruments for more routine, nondestructive analysis. S-CT with Fresnel zone plate (FZP) has emerged as a true nanometerscale method for 3D imaging, but is only appropriate for extremely high value samples [e.g., 14].

Chondritic meteorites are composed primarily of 0.1 to $1.0 \mathrm{~mm}$ diameter igneous objects (chondrules) and aggregates of refractory minerals (Ca-, Al-rich inclusions, CAIs), the oldest objects formed in the solar system, all surrounded by a matrix of fine-grained mineral dust in varying proportions. The sizes and densities of these objects are critical constraints on astrophysical theories of planetary accretion. MicroCT imaging of individual chondrules requires $\sim 1 \mu \mathrm{m} /$ voxel edge resolution, while resolutions finer than $\sim 20 \mu \mathrm{m} /$ voxel edge provide useful images for larger whole meteorite stones. We therefore restrict our S-CT work to samples with $\sim 1 \mathrm{~cm}$ or smaller maximum width, and MicroCT work to samples $<\sim 3 \mathrm{~cm}$ width. The monochromatic S-CT beam is $<45 \mathrm{KeV}$, while currents up to $140 \mathrm{KeV}$ are used with MicroCT. The AMNH purchased a GE Phoenix VtomeX S240 dual tube CT instrument in 2010 for investigating chondritic meteorites, among a huge range of applications in the natural sciences.

MicroCT imaging is a fundamental first step to chemical and isotopic analyses of cut 2D surfaces of meteorites. Figure 1 illustrates the close correspondence between 2D RGB mosaic and back-scattered 
electron (BSE) images, and a single virtual MicroCT slice through the pre-cut volume of the primitive ordinary chondrite Semarkona (LL3.0). Careful trackng is required, in keeping track of geometric relationships between many such slices, both actual and virtual, through a single $\sim 1 \mathrm{~cm}^{3}$ volume of rock.

Experience with meteorites informs efforts to image in 3D the tracks formed by hypervelocity collection of comet particles using laser scanning confocal microscopy (LSCM) at $\sim 80$ x 80 x $300 \mathrm{~nm}$, complemented by 2D or stereo synchrotron X-ray fluorescence mapping (S-XRF) at $2 \mu \mathrm{m} /$ pixel [15]. In this application, 3D optical imaging and S-XRF inform subsample selection, provide composition information, and will inform modeling of the collection process and original impactor characteristics.

The curation of complementary 3D and 2D data on extraterrestrial samples is challenging but essential for initial characterization, guided sub-sampling, and the return of maximum information.

\section{References:}

[1] DS Ebel and ML Rivers, Meteoritics and Planetary Science 42 (2007), p. 1627-1646.

[2] JM Friedrich et al., Earth and Planetary Science Letters 275 (2008), p. 172-180.

[3] JM Friedrich et al., Planetary and Space Science 56 (2008), p. 895-900.

[4] DS Ebel et al., Meteoritics and Planetary Science 43 (2008), p. 1725-1740.

[5] MR Sasso et al., Meteoritics and Planetary Science 44 (2009), p. 1743-1753.

[6] P Jenniskens et al., Science 338 (2012), p. 1583-1587.

[7] www.sciencemag.org/cgi/content/full/338/6114/1583/DC1

[8] http://digitallibrary.amnh.org/dspace/handle/2246/6408

[9] SW Wallace, DS Ebel, and MG Hill, Lunar and Planetary Science XLIV (2013), Abs 2297.

[10] D Heymann et al., Meteoritics 20 (1985), p. 559-569.

[11] GJ Flynn et al., Lunar and Planetary Science XXX1 (2000), Abs \#1893.

[12] A Tsuchiyama et al., Geochemical Journal 36 (2002), p. 369-390.

[13] A Tsuchiyama et al., Science 333 (2011), p. 1125-1128.

[14] T Nakamura et al., Science 321 (2008), p. 1664-1667.

[15] AJ White, DS Ebel, and M Greenberg, Lunar and Planetary Science XLIV (2013), Abs 1630.

[16] The authors acknowledge funding from NASA COS grant NNX10AI42G (DSE) and NASA OSS grant NNX10AH336 (JMF). Use of the Advanced Photon Source, an Office of Science User Facility operated for the U.S. Department of Energy (DOE) Office of Science by Argonne National Laboratory, was supported by the U.S. DOE under Contract No. DE-AC02-06CH11357.
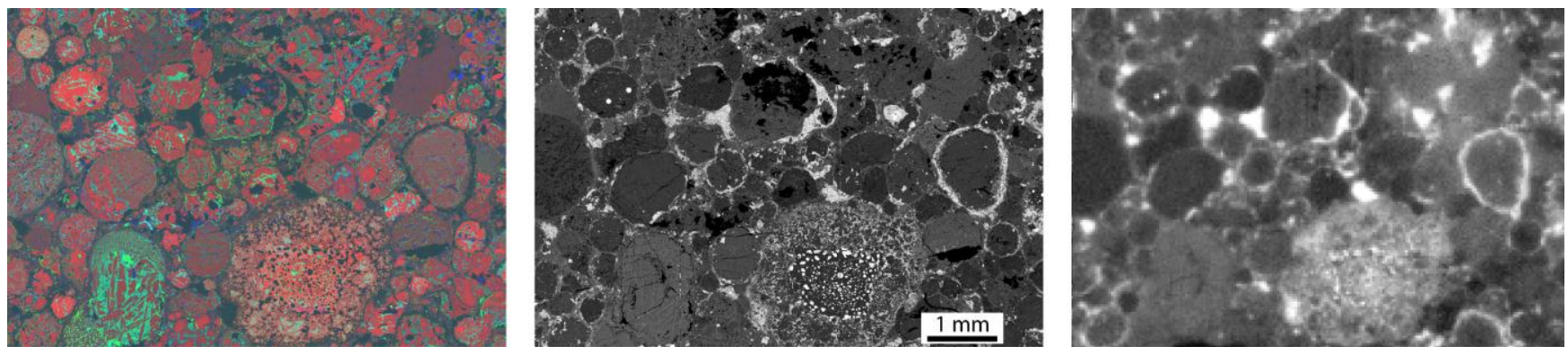

Figure 1. Semarkona (LL3.0) chondrite. Left: R-G-B = Mg-Ca-Al EPMA x-ray intensity composite mosaic. Center: EMPA BSE image. Right: S-CT slice (x_0010) of AMNH \#4128, Sem4128-t1-ps1A. EPMA is at $5 \mu \mathrm{m} /$ pixel, S-CT at $17.07 \mu \mathrm{m} /$ voxel edge. Bright pixels in BSE and S-CT are metal. 